EPJ Web of Conferences 64, 02003 (2014)

DOI: $10.1051 /$ epjconf/ 20146402003

(C) Owned by the authors, published by EDP Sciences, 2014

\title{
A multi-model approach to X-ray pulsars
}

\section{Connecting spectral and timing models to pin down the intrinsic emission characteristics of magnetized, accreting neutron stars}

\author{
G. Schönherr ${ }^{1,2, a}$, F. Schwarm ${ }^{2}$, S. Falkner ${ }^{2}$, P. Becker ${ }^{3}$, J. Wilms $^{2}$, T. Dauser ${ }^{2}$, M. T. Wolff ${ }^{4}$, K.
} Wolfram $^{3}$, B. West ${ }^{3}$, K. Pottschmidt ${ }^{5}$, P. Kretschmar ${ }^{6}$, C. Ferrigno ${ }^{7}$, D. Klochkov ${ }^{8}$, O. Nishimura ${ }^{9}$, I. Kreykenbohm ${ }^{2}$, I. Caballero ${ }^{10}$, R. Staubert ${ }^{8}$, and The MAGNET collaboration

${ }^{1}$ Leibniz-Institut für Astrophysik Potsdam (AIP), An der Sternwarte 16, 14482 Potsdam, Germany

${ }^{2}$ Dr. Remeis-Sternwarte \& ECAP, Sternwartstr. 7, 96049 Bamberg, Germany

${ }^{3}$ George Mason University, 4400 University Drive, Fairfax, VA 22030, USA

${ }^{4}$ High Energy Space Environment Branch, Space Science Division, Naval Research Laboratory, Washington DC 20375, USA

${ }^{5}$ CRESST, University of Maryland Baltimore County and NASA's Goddard Space Flight Center, Greenbelt, MD 20771, USA

${ }^{6}$ European Space Astronomy Centre (ESA/ESAC), Science Operations Dept., P.O. Box 78, 28691 Villanueva de la Cañada, Madrid, Spain

${ }^{7}$ INTEGRAL Science Data Centre, Université de Genève, Chemin d'Écogia 16, 1290 Versoix, Switzerland

${ }^{8}$ Institut für Astronomie und Astrophysik, Sand 1, Abt. Astronomie, Universität Tübingen, Germany

${ }^{9}$ Department of Electronics and Computer Science, Nagano National College of Technology, 716 Tokuma, 381-8550 Nagano, Japan

${ }^{10}$ CEA Saclay, CNRS/CEA/Université P. Diderot, F-91191 Gif sur Yvette, France

\begin{abstract}
The emission characteristics of X-ray pulsars are governed by magnetospheric accretion within the Alfvén radius, leading to a direct coupling of accretion column properties and interactions at the magnetosphere. The complexity of the physical processes governing the formation of radiation within the accreted, strongly magnetized plasma has led to several sophisticated theoretical modelling efforts over the last decade, dedicated to either the formation of the broad band continuum, the formation of cyclotron resonance scattering features (CRSFs) or the formation of pulse profiles. While these individual approaches are powerful in themselves, they quickly reach their limits when aiming at a quantitative comparison to observational data. Too many fundamental parameters, describing the formation of the accretion columns and the systems' overall geometry are unconstrained and different models are often based on different fundamental assumptions, while everything is intertwined in the observed, highly phase-dependent spectra and energy-dependent pulse profiles. To name just one example: the (phase variable) line width of the CRSFs is highly dependent on the plasma temperature, the existence of B-field gradients (geometry) and observation angle, parameters which, in turn, drive the continuum radiation and are driven by the overall two-pole geometry for the light bending model respectively. This renders a parallel assessment of all available spectral and timing information by a compatible across-models-approach indispensable. In a collaboration of theoreticians and observers, we have been working on a model unification project over
\end{abstract}

ae-mail: g.schoenherr@aip.de

This is an Open Access article distributed under the terms of the Creative Commons Attribution License 2.0, which permits unrestricted use, distribution, and reproduction in any medium, provided the original work is properly cited. 
the last years, bringing together theoretical calculations of the Comptonized continuum, Monte Carlo simulations and Radiation Transfer calculations of CRSFs as well as a General Relativity (GR) light bending model for ray tracing of the incident emission pattern from both magnetic poles. The ultimate goal is to implement a unified fitting model for phase-resolved spectral and timing data analysis. We present the current status of this project.

\section{Introduction}

The neutron stars in accreting X-ray pulsars possess very strong magnetic fields of the order of $10^{12}$ Gauss that dominate the accretion process: For all accretion types (e.g. wind accretion, Be accretion, or Roche lobe overflow), the magnetospheric coupling of the accretion flow to the field lines governs the formation of the accretion columns and hence determines the geometrical and physical setting for the regions in which the observed X-ray radiation is ultimately generated. The observed radiation shows a rich morphology both in spectral and timing analyses. Cyclotron resonance scattering features have been observed in the spectra of more than a dozen X-ray pulsars. Most sources have highly complex energy-dependent and often multi-peaked pulse profiles. The analysis of observational data, however, to date has been widely restricted to fitting phenomenological models. A complete physical model is still lacking. Progress has been made during the past years in developing fitting routines based on physical models with a focus on either the continuum or on cyclotron line features (cyclomc ${ }^{1}$ : Schönherr \& Wilms [1], bwmod ${ }^{1}$ : Ferrigno, Becker, Wolff [2, 3], compmag: Farinelli et al. [4], cyclomod ${ }^{1}$ : Schwarm, Schönherr, Wilms). Striving for a combination and meaningful parametrization of the two individual models bwmod for the continuum and cyclomod for the cyclotron lines has been one starting point of our multi-model project. It is important to keep in mind, however, that these models have been designed to mimic the intrinsic radiation from one accretion column at one magnetic pole. In order to properly model observed X-ray pulsar spectra, it is necessary to consider the observable radiation from both poles far from the neutron star as a function of energy and rotational phase, and to integrate it for comparison to phase-averaged spectra. The sketch in Figure 2 illustrates this geometrical problem of discriminating between contributions from both poles, which has been investigated in more detail by different authors for several sources in the past years based on a pulse profile decomposition approach by U. Kraus et al. [5]. For early works on the formation of X-ray pulsar spectra including light bending effects we refer to [6] and references therein.

In Section 2 we sketch our general assumptions on the geometric and physical characteristics of the accreting system. In Section 3 we present the individual model components and how they are coupled together into one model. In Section 4 we first show our results with examples of synthetic observable spectra and pulse profiles and finally we discuss our conclusions on the present status of this new model.

\section{Physical setup and parameters}

A big challenge in our approach to a unified fitting model for X-ray pulsars is the definition of a parameter space which should be at the same time large enough to model a variety of possible physical

\footnotetext{
${ }^{1}$ the model can be obtained from the authors.
} 


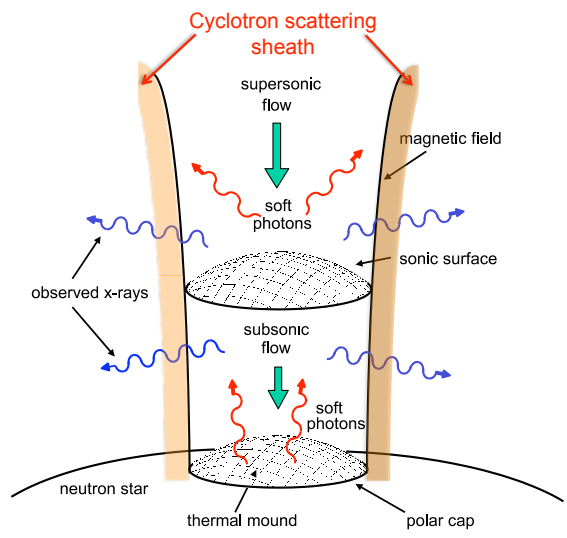

Figure 1. Illustration of the formation of the intrinsic radiation at the base of the accretion column using a hybrid model approach for the continuum generation and for CRSF formation in a photosphere around the optically thick core. The figure has been adapted from Fig.1 in [2].

setups - reflecting the few constraints we have on the magnetospheric accretion process for High-Mass X-ray Binaries - and small enough to allow for building and running a high-quality fitting routine.

We consider a neutron star with a strong magnetic field accreting matter onto two magnetic poles, representing a (possibly deformed) dipole. The location of the magnetic poles is in principle free, as there are no available reference calculations predicting their location and shape on the neutron star surface, for example, from 3D MHD simulations. The axes through the poles are offset by angles $i_{1}, i_{2}$ with respect to the rotational axis of the neutron star and the poles can have an offset in azimuthal angle. The whole system is observed under an inclination $i$. The intrinsic X-ray emission is assumed to be formed within the optically thick core deep within the central part of the accretion column [2,7] (see Figure 1). The X-ray photons diffuse outward while further interacting via resonant scattering processes with the plasma electrons. Photons with energies close to the cyclotron resonances induce transitions between the magnetically quantized, quasi-harmonically spaced Landau energy levels of the electrons. Due to the resonant nature of the cross sections, absorption-like features are generated in the spectra and a strong redistribution of the escaping photons' angles with respect to the magnetic field occurs. The inner core is characterized by a moderate continuum optical depth $(\tau \approx 1-10)$ while the cyclotron lines are assumed to form in a thin sheath of small Thomson optical depth $\left(\tau \approx 10^{-4}-10^{-3}\right)$. Further central model parameters describing the emission regions are assumed functions of height in the column (h) and are the magnetic field structure $B(h)$, the density and velocity profiles $(\rho(h), v(h))$, and the parallel electron temperature $k T_{e}(h)$. As a starting point we assume the simplified picture of a static plasma with constant $B, \rho, k T_{e}$ within the emitting volume in order to keep the number of free parameters small. The influence of magnetic field, temperature and density gradients on the continuum (e.g. [2,7]) and on the cyclotron line shapes (e.g. [8]) have been discussed previously; such gradients shall also be investigated with our unified model in the future. For a discussion of the effects of a non-static plasma on the observed flux within our model, see $[9,10]$.

\section{Go multi model}

In the following the individual model components are briefly described. For more details we refer to the original publications. 


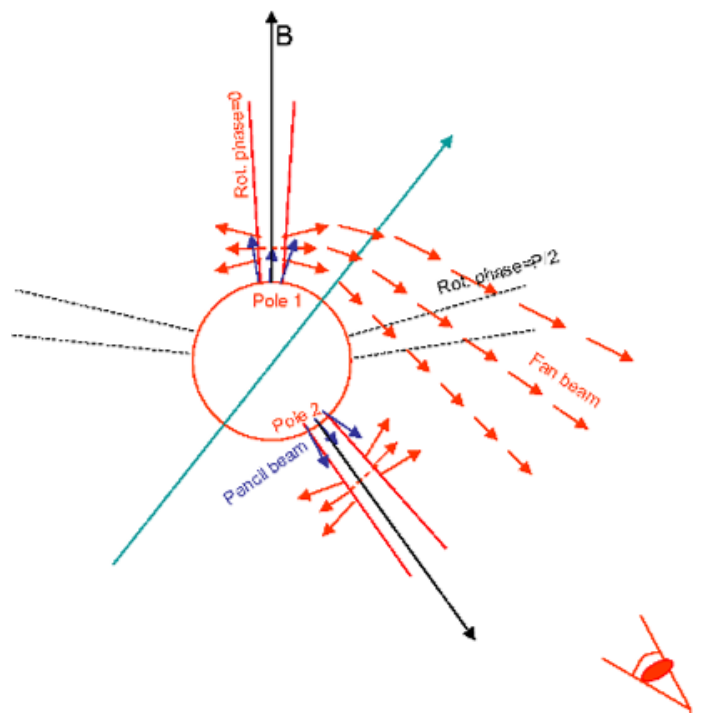

Figure 2. (Simplified) illustration of the effect of mixing of radiation from both poles for a far observer. Gravitational light bending can for instance lead to the simultaneous observation of fan and pencil beam components from opposite poles, rendering those components indistinguishable in the phase-averaged spectrum.

\subsection{Continuum}

We start by generating an X-ray continuum source at one pole using the model by Becker \& Wolff [2]. These authors have obtained the analytical solution for the spectral formation process, considering bulk and thermal Comptonization of radiation from a monochromatic source as a function of height in the accretion column. They calculate the total X-ray pulsar spectrum by convolution with bremsstrahlung and cyclotron sources distributed along the column, and with a black body source at the surface of the neutron star.

\subsection{Cyclotron lines}

Second, the cyclotron resonance scattering features (short: "cyclotron lines") are generated by convolution of the X-ray continuum with a Green's functions database which is provided by pre-calculated Monte Carlo simulations from an implementation by Schwarm et al. following Araya \& Harding (1999) and Schönherr et al. (2007) [1, 9, 11]. The Monte Carlo simulations use the fully relativistic QED cross sections [12-14] but average over polarization for the sampling of the scattering process. The scattering profiles have also been verified in comparison to the ones used by $\mathrm{O}$. Nishimura for his Feautrier model (priv. comm.). The highly angle-dependent, resonant cross sections cause a strong angle-dependence in the emergent radiation. We thus obtain the intrinsic emitted flux at one magnetic pole as a function of energy and angle measured with respect to the magnetic field.

\subsection{Light bending and pulse profiles}

Third, we apply a numerical routine implemented by Falkner et al. [15] to calculate the effects of General Relativity on the photons emerging from the very close vicinity of the compact object and to transform the angle-dependent intrinsic flux to a phase-dependent observed flux. The routine samples the neutron star and accretion column surface numerically by dividing that surface into a large number of triangular surface elements. This technique can in principle be adapted to any two-pole geometry 


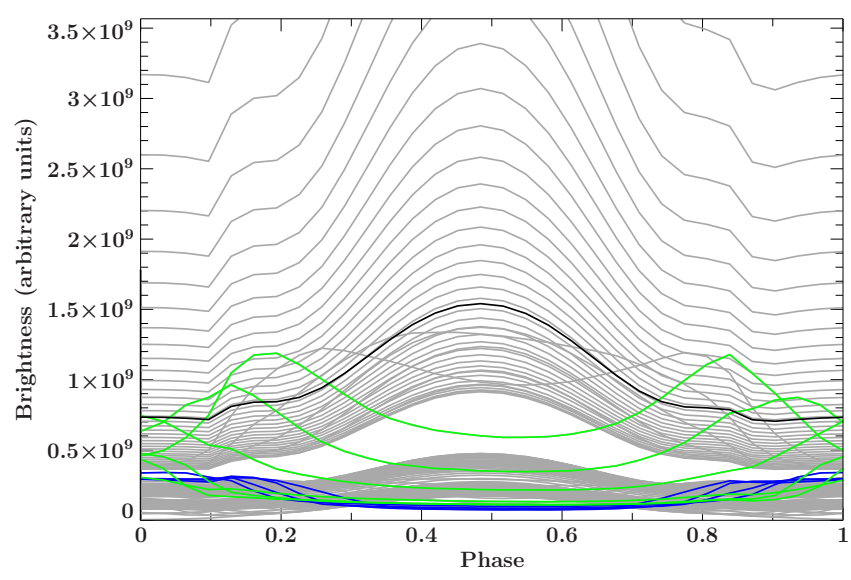

Figure 3. Evolution of an example pulse profile over energy (grey lines: energy increasing from top to bottom). The energy-averaged pulse profile is shown as a black line for comparison (flux not to scale). A significant change in morphology can occur near the cyclotron energies. A single peaked profile can for instance change into a multi-peaked profile as shown here. The pulse profiles for photons escaping near the fundamental cyclotron energy are highlighted in green (just below $E_{\text {cyc }}$ ) and blue (just above $E_{\text {cyc }}$ ).

with arbitrary emission patterns. Integrating over the angle-dependent intrinsic fluxes from both poles of a previously chosen two-pole and observing geometry for each phase bin, we obtain the rotation phase-dependent observed total spectra and energy-dependent pulse profiles.

\section{Results and Conclusions}

Figures 3 and 4 show preliminary results of complex pulse profiles and spectra for rather simplified physical and geometrical settings. Some energy-resolved pulse profiles have striking deviations in their morphology near the cyclotron energies compared to the average pulse profile. For instance, a single, broad pulse peak can split into multiple peaks (Figure 3) and apparent phase lags can occur at the cyclotron line energies relative to the average pulse profile. The observability of such trends is strongly governed by both the physical flow parameters and the observing geometry as we will discuss in greater detail in a forthcoming publication [10]. They can emerge even for rather simple geometries of the individual emitting regions at the magnetic poles.

An example of a particularly strong variability of the cyclotron line shapes with phase is shown in Figure 4. For this example we have assumed two antipodal accretion mounds which both emit radiation as a combination of fan and pencil beam patterns. The overall spectral shape and the amount of expected variability (which can also be very little) strongly depend on all geometric input parameters, as is expected from the strong angular dependence of the cyclotron resonant scattering cross sections, and will be the subject of further investigation.

Already at this first stage of modelling, it is clear that the interpretation of observable features in $\mathrm{X}$-ray pulsars requires a parallel assessment of phase-dependent spectra and energy-dependent pulse profiles. With our multi-model approach we can predict their shapes self-consistently to test and to disentangle physical and geometrical assumptions. Thus generated synthetic fluxes can qualitatively reproduce certain variability characteristics of observed phase and energy dependent fluxes. The next steps will be to systematically investigate and expose the relative impact of the various physical and 


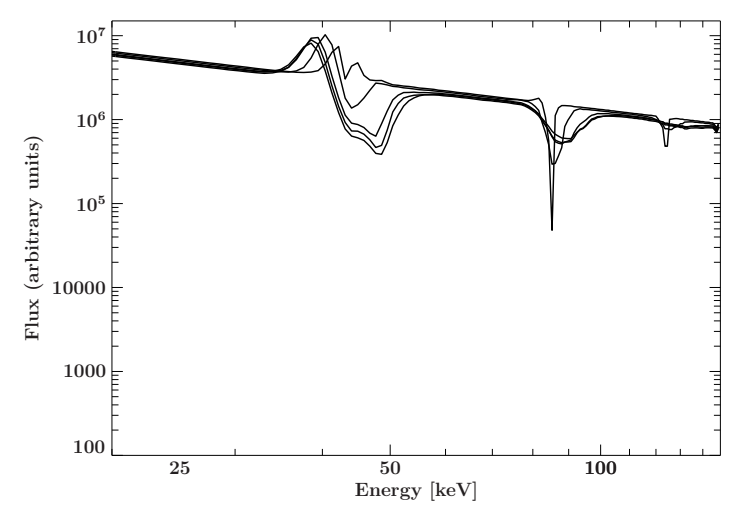

Figure 4. Example for a particularly strong variability of the cyclotron line shapes with spin phase. Both the sidewalls and the top of the mound were assumed to contribute to the intrinsic emission from antipodally located accretion mounds. The input continuum has been assigned a simple powerlaw without high-energy cutoff for better illustration of the higher harmonics. The cyclotron lines in the intrinsic spectra have been calculated from Green's functions corresponding to a magnetic field strength of $4 \times 10^{12}$ Gauss, a parallel electron temperature of $k T_{e}=3 \mathrm{keV}$ and an optical depth of $\tau=10^{-3}$.

geometrical free parameters (also allowing for parameter gradients within the line-forming region), the comparison to key observables, and ultimatively to fit the theoretical $2 \mathrm{D}$ phase-energy matrices quantitatively to the observed data of a real source.

\section{Acknowledgements}

We thank the International Space Science Institute, Bern, for hosting two international team meetings about The physics of the accretion column of X-ray pulsars in 2011/2012 to start this collaborative effort. MTW is supported by the NASA Astrophysics Data Analysis Program.

\section{References}

[1] G. Schönherr, J. Wilms, P. Kretschmar, I. Kreykenbohm, A. Santangelo, R.E. Rothschild, R. Staubert, W. Coburn, Astron. \& Astrophys. 472, 353 (2007)

[2] P.A. Becker, M.T. Wolff, Astrophys. J. 654, 435 (2007)

[3] C. Ferrigno, P.A. Becker, A. Segreto, T. Mineo, A. Santangelo, Astron. \& Astrophys. 498, 825 (2009)

[4] R. Farinelli, C. Ceccobello, P. Romano, L. Titarchuk, Astron. \& Astrophys. 538, A67 (2012)

[5] U. Kraus, H.P. Nollert, H. Ruder, H. Riffert, Astrophys. J. 450, 763 (1995)

[6] P. Mészáros, W. Riffert, Astrophys. J. 327, 712 (1988)

[7] P.A. Becker, M.T. Wolff, Astrophys. J. 630, 465 (2005)

[8] O. Nishimura, Astrophys. J. 672, 1127 (2008)

[9] F.W. Schwarm, G. Schönherr, J. Wilms, A new simulation of cyclotron lines: The effect of bulk velocity, in this volume: Proc. Physics at the Magnetospheric boundary (2013)

[10] G. Schönherr, F. Schwarm, S. Falkner, T. Dauser, C. Ferrigno, M. Kühnel, D. Klochkov, P. Kretschmar, P. Becker, M. Wolff et al., Astron. \& Astrophys. (2013), submitted

[11] F.W. Schwarm, Diploma thesis, University of Erlangen-Nuremberg, Erlangen (2010)

[12] R. Sina, Ph.D. thesis, University of Maryland (1996)

[13] A.K. Harding, J.K. Daugherty, Astrophys. J. 374, 687 (1991)

[14] R.A. Araya, A.K. Harding, Astrophys. J. 517, 334 (1999)

[15] S. Falkner, T. Dauser, M. Falanga, J. Wilms, Acta Polytechnica, (2013), submitted 